\title{
Genome sequencing and protein domain annotations of Korean Hanwoo cattle identify Hanwoo-specific immunity-related and other novel genes
}

Kelsey Caetano-Anolles ${ }^{2}$, Kwondo Kim ${ }^{1}$, Woori Kwak ${ }^{1,3}$, Samsun Sung ${ }^{3}$, Heebal Kim ${ }^{1,2,3}$, Bong-Hwan Choi ${ }^{4}$ and Dajeong Lim $^{4^{*}}$

\begin{abstract}
Background: Identification of genetic mechanisms and idiosyncrasies at the breed-level can provide valuable information for potential use in evolutionary studies, medical applications, and breeding of selective traits. Here, we analyzed genomic data collected from 136 Korean Native cattle, known as Hanwoo, using advanced statistical methods.

Results: Results revealed Hanwoo-specific protein domains which were largely characterized by immunoglobulin function. Furthermore, domain interactions of novel Hanwoo-specific genes reveal additional links to immunity. Novel Hanwoo-specific genes linked to muscle and other functions were identified, including protein domains with functions related to energy, fat storage, and muscle function that may provide insight into the mechanisms behind Hanwoo cattle's uniquely high percentage of intramuscular fat and fat marbling.

Conclusion: The identification of Hanwoo-specific genes linked to immunity are potentially useful for future medical research and selective breeding. The significant genomic variations identified here can crucially identify genetic novelties that are arising from useful adaptations. These results will allow future researchers to compare and classify breeds, identify important genetic markers, and develop breeding strategies to further improve significant traits.
\end{abstract}

Keywords: Cattle, Hanwoo, Genome sequencing, Protein domain, Unaligned read assembly, DNA-Seq

\section{Background}

Hanwoo is a Korean native taurine breed of cattle that has been around since $2000 \mathrm{BC}$. Although their original primary purpose was to serve as farming and transportation cattle, the rapid growth of the Korean economy that occurred in the 1960's and its associated food demands led to this breed being used as a main source of meat [1]. Since then, the demand for this product in Korea has skyrocketed. This is due to the high percentage of fat marbling in Hanwoo meat, a characteristic that is unique to the breed. Hanwoo loin muscles have approximately $24 \%$ intramuscular fat content [2]. The quality and price of meat is often determined by fat marbling.

\footnotetext{
* Correspondence: lim.dj@korea.kr

${ }^{4}$ Animal Genomics \& Bioinformatics Division, National Institute of Animal Science, RDA, 77 Chuksan-gil, Kwonsun-gu, Suwon 441-706, Republic of Korea

Full list of author information is available at the end of the article
}

Consequently, one of the main goals of the meat production industry worldwide is to increase the incidence of this trait [2]. Given this focus, several studies have investigated gene expression patterns with the primary goal of determining which genes are responsible for Hanwoo-specific high fat concentration [3-7].

Here we gathered genomic data from 136 Hanwoo catthe that we analyzed using advanced statistical methods. We show that investigation of the genome of this unique set of cattle individuals with the general goal of identifying breed-level idiosyncrasies can provide valuable information for potential use in evolutionary studies, medical applications, and breeding of selective traits. The goal is to enhance our understanding of characteristics of beef cattle breeds with unique adaptations and beneficial traits that have not yet been well elucidated. This would make it possible to selectively breed for these traits in 
other breeds of cattle worldwide to improve meat quality and revolutionize the field of meat production.

\section{Methods}

Alignment of unaligned reads for the detection for novel genes using the Hanwoo whole genome

Blood samples for whole genome sequencing were obtained from 136 Korean beef cattle (Hanwoo) individuals reared at the Hanwoo Improvement Center of the National Agricultural Cooperative Federation (Seosan, Chungnam, Korea). Indexed shotgun paired-end (PE) libraries with $500 \mathrm{bp}$ average length inserts were generated from these samples using the TruSeq Nano DNA Library Prep Kit (Illumina, USA) following the standard Illumina sample-preparation protocol. Briefly, $200 \mathrm{ng}$ of gDNA was fragmented using a Covaris M220 focused-ultrasonicator (Woburn, MA, USA) to produce fragments with a median size of $\sim 500 \mathrm{bp}$. The fragmented DNA was subjected to end repair, A-tailing, and indexed adapter ligation ( 125 bp adapter). Adapter-ligated DNA of 550 to $650 \mathrm{bp}$ in length was amplified using PCR for 8 cycles. The size-selected libraries were analyzed using the Agilent 2100 Bioanalyzer (Agilent Technologies) to determine the size distribution and to check for adapter contamination. The resulting libraries were sequenced using the Illumina HiSeq 2500 (2x125bp paired-end sequences) and NextSeq500 (2x150bp paired-end sequences) Next-Gen sequencers.

The bioinformatics pipeline used in this study is described in Figs. 1 and 2. Quality control for per-base quality of reads and removal of potential adaptor sequences was performed using fastQC v0.11.4 [8] and Trimmomatic v0.36 [9] software (seed mismatches:2, palindrome clip threshold:30, simple clip threshold:10,
LEADING:10, TRAILING:10, MINLEN:80), respectively. Then, high-quality sequence reads were mapped to the Bos taurus reference genome (UMD 3.1) using Bowtie2.2.6 [10] with default settings in order to extract unaligned reads. Removal of duplicate reads was performed using Picard (ver 1.06) and indexing, sorting, and unaligned read extraction was performed using Samtools v1.3.1 [11]. GATK v3.4.46 [12-14] was used for local realignment and recalibration of the alignment (blue boxes on the pipeline figure; Fig. 1). A summary of sequencing data is provided in Additional file 1: Table S1.

Since we are interested in information originating from the sample itself and not detected from the reference sequence, we created an assembled genome at the scaffold level to discover whether unaligned reads actually constitute functional units (genes) on their own genome. This scaffold was created from one randomly selected sample from our pool of samples. The Broad Institute's stand-alone ALLPATHS-LG fragment read error correction module $[15,16]$ was used for error correction as a precursor to de novo assembly. De novo assembly was performed using an Iterative De Bruijn Assembler of Uneven Depth (IDBA_UD: [17, 18], an iterative De Bruijn graph de novo assembler for short reads sequencing data that utilizes paired-end reads to assemble highly uneven low-depth regions. This tool is useful for optimizing the length gap problem and iterating different $\mathrm{K}$-mer length (green boxes on the pipeline figure; Fig. 1).

For unaligned read alignments, we extracted reads for each sample that was not aligned to the reference genome. Using the extracted unaligned reads (blue boxes on the pipeline figure; Fig. 1) and the assembled scaffold-level genome (green boxes on the pipeline figure; Fig. 1) of each sample, alignment of unaligned

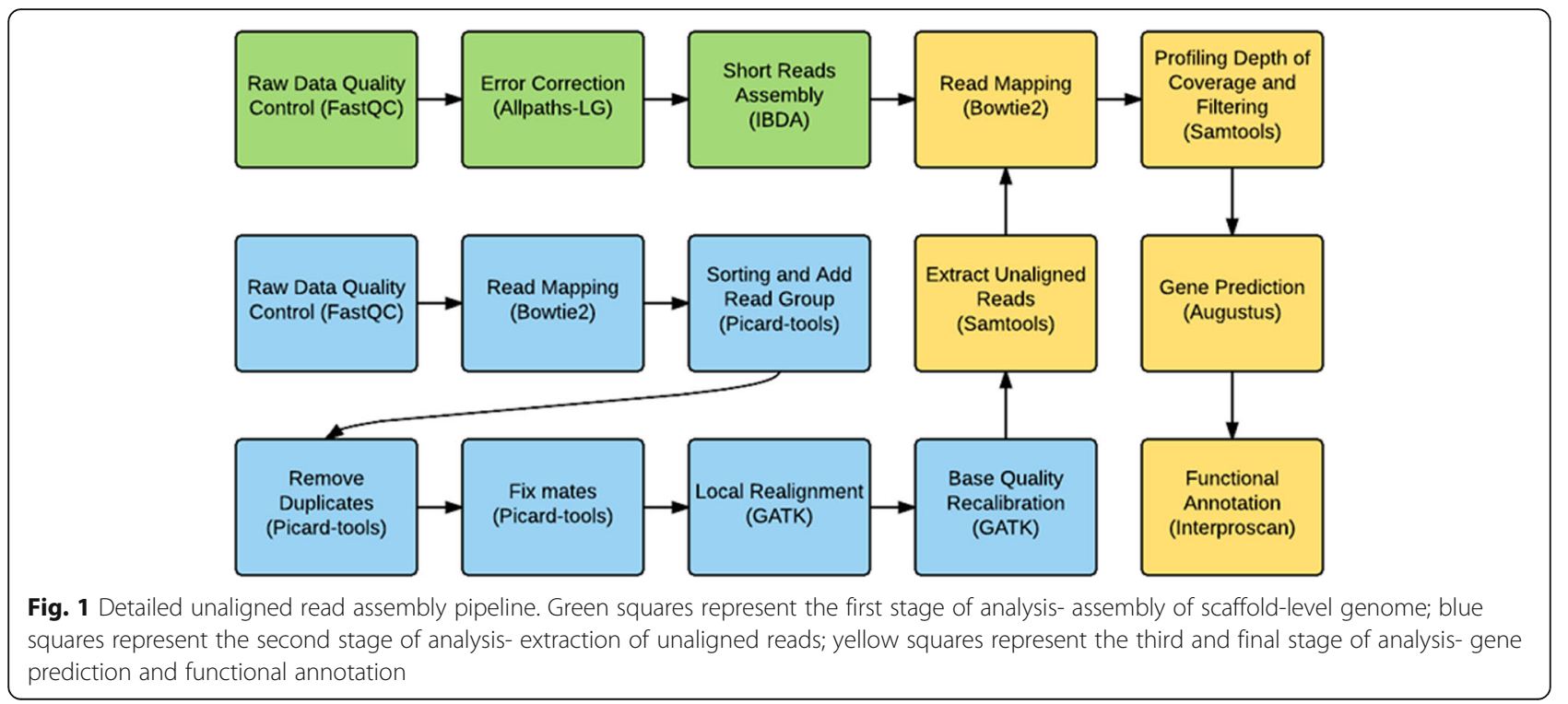




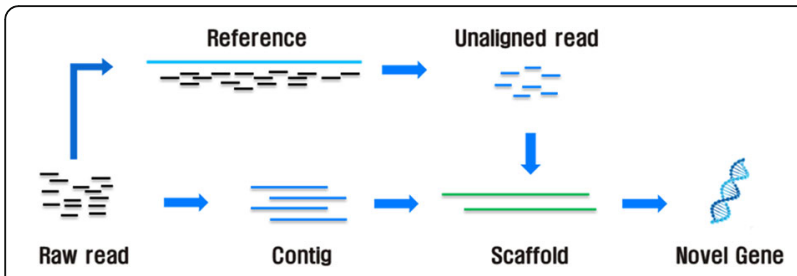

Fig. 2 Simplified pipeline of unaligned read assembly

reads to the scaffold was carried out using Bowtie2 (remapping). The identified remapped sequences throughout the sequence were assumed to represent Hanwoo-specific sequences. These resulting regions constitute regions that are distinctive from the reference. We performed depth profiling to diminish the possibility of false positives. We identified scaffolds containing locations meeting our depth cutoff of 10x (an arbitrary cutoff selected for result filtering), and used the collected scaffolds for gene prediction using the gene prediction program Augustus 3.1.0. Out of the resulting 614 predicted genes, we extracted protein sequences covered by unaligned reads with at least depth of $10 \times$.

The resulting total of 283 protein sequences were cross-referenced against the Pfam database of protein families (pfam.xfam.org; [19]) using the protein domain detection program InterProScan-5.15-54.0 in order to identify protein domains affiliated with those areas of the genome. In order to assign meaning and infer the function of these domains, we searched for these identified domains within DOMINE (http://domine.utdallas.edu/cgi-bin/Domine), a database of known and predicted protein domain interactions [20, 21]. Using Interpro [22], we obtained GO (Gene Ontology) Cellular Component (CC), Molecular Function (MF), and Biological Process (BP) terms for each individual domain [23]. Next, gene ontology results were summarized and visualized with the online tool REVIGO (http://revigo.irb.hr; [24]) to better interpret our results. Next, using REVIGO's Interactive Graph tool [24] and exporting results into the Cytoscape software package [25], we created a graph-based visualization of the identified terms for each GO category.

Using the above described methodologies and annotations we were able to align and map genome sequences as well as predict genes that may be related to Hanwoo-specific characteristics.

\section{Results and discussion}

\section{Research objectives and genome build summary}

Our main research objectives included: (1) Assembling and mapping unaligned reads in order to identify and predict genes in Hanwoo cattle; (2) Cross-referencing results against a comprehensive protein domain database in order to identify protein domains affiliated with those areas of the genome; and (3) Mining the uncovered genes and associated domains to identify important gene functions and networks involved in positive traits.

A summary of representative reference genome builds via short read assembly is presented in Table 1 . We mapped unaligned reads against the reference genome and extracted information to a depth of $10 \times$ (meaning that each base was sequenced an average of 10 times). We predicted a total of 614 gene regions using scaffolds containing locations higher than depth of $10 \times$. Of the 614 genes, 283 genes were covered by unaligned reads with at least depth of $10 \times$.

Cross-referencing of protein sequences from the 283 genes against the Pfam database identified associated protein domains covering a total of 168 scaffolds. Overall, 311 Pfam protein domains were identified when using data filtered for sequences with an average mapped base depth coverage of less than 10x. These numbers suggest that there was more than one affiliated domain identified for some gene regions. Due to space limitations, Table 2 lists significantly identified (E- value $<1 \mathrm{XE}-100)$ Pfam protein family domain analysis results. An extended list of significantly identified Pfam domains with $E$-value $<1 \mathrm{E}-40$ is presented in Additional file 2: Table S2.

\section{Hanwoo-specific genes linked to immunity}

A number of domains were largely characterized by immune system function. Selected immune system-related genes are shown in Table 3. Six of the seven domains shown are associated to the immunoglobulin function, while the remaining domain is associated with the interferon group of signaling proteins, which is crucial for the immune system response as well.

The interferon-alpha/beta receptor is a cell surface receptor made up of one chain with two subunits, IFNAR1 and IFNAR2. The interferon receptors have antiviral,

Table 1 Summary of the results of representative reference genome build via short read assembly ( $>=1 \mathrm{~kb})$

\begin{tabular}{llll}
\hline & & Base pairs & Percent (\%) \\
\hline Number of scaffolds & & 295,265 & 100 \\
Residue counts & A & $701,475,984$ & 29.24 \\
& C & $498,799,879$ & 20.79 \\
& G & $498,441,379$ & 20.78 \\
& T & $692,999,844$ & 28.89 \\
& N & $7,063,142$ & 0.29 \\
Sequence lengths & Total & $2,398,780,228$ & 100 \\
& Minimum & 1000 & \\
& Maximum & 136,625 & \\
& Average & 8124.16 & \\
& N50 & 13,528 & \\
\hline
\end{tabular}


Table 2 Significantly identified ( $E$ - value <1XE-100) Pfam protein family domain analysis results

\begin{tabular}{|c|c|c|c|c|c|c|c|}
\hline Gene name & Length & Source & Accession & Description & Start & Stop & E-value \\
\hline scaffold_2197.g59.t1 & 581 & Pfam & PF00063 & Myosin head (motor domain) & 30 & 575 & 5.60E-207 \\
\hline scaffold_1285.g30.t1 & 417 & Pfam & PF15718 & Domain of unknown function (DUF4673) & 116 & 412 & 4.50E-154 \\
\hline scaffold_6851.g129.t1 & 391 & Pfam & PF03028 & Dynein heavy chain and region D6 of dynein motor & 2 & 390 & 1.50E-120 \\
\hline scaffold_13817.g209.t1 & 758 & Pfam & PF01403 & Sema domain & 59 & 467 & 2.30E-117 \\
\hline scaffold_29068.g344.t1 & 348 & Pfam & PF16021 & Programmed cell death protein 7 & 33 & 344 & $3.00 \mathrm{E}-114$ \\
\hline scaffold_15941.g224.t1 & 887 & Pfam & PF04849 & HAP1 N-terminal conserved region & 1 & 249 & 2.60E-108 \\
\hline scaffold_5769.g113.t1 & 246 & Pfam & PF00244 & 14-3-3 protein & 5 & 238 & 3.60E-107 \\
\hline scaffold_1936.g56.t1 & 564 & Pfam & PF08235 & LNS2 (Lipin/Ned1/Smp2) & 300 & 525 & 1.30E-104 \\
\hline
\end{tabular}

antiproliferative, and immunomodulatory functions, as well as being highly involved in pregnancy $[26,27]$. Interferon- $\tau$, a type I interferon, has been shown to prevent a return to ovarian cyclicity after conception to ensure the continuation of the pregnancy in ruminant ungulate species; this interferon appears to be the main factor responsible for prevention of degradation of the corpus luteum $[28,29]$.

In addition to these reproductive roles, this receptor is responsible for binding type 1 interferons interferon- $\alpha$ and $-\beta$ and activating the JAK-STAT signaling pathway, which is associated with DNA-transcription and the expression of genes related to immunity, proliferation, and differentiation, among others [30]. The JAK-STAT pathway has primary functions related to immunity. In fact, drug therapies that aim to turn down the immune response of the body and modulate host responses to disease and infection target this pathway [31]. The expression of the interferon group of signaling proteins in our Hanwoo cattle samples suggests that Hanwoo may have breed-specific immune system functions that are not yet well understood.

Our analysis also identified associated protein domains which are largely characterized by the immunoglobulin function. These results are particularly salient given the significance of these kinds of results for medical research and selective breeding. The bovine immune system has been a topic of interest to researchers for quite some time now, mainly due to two reasons [32]. The first is that an understanding of the evolution and expression of mammalian immune system genes has important implications for human health. Bovine antibodies have been of particular interest, as they exhibit prophylactic and therapeutic properties in response to several human and animal infectious diseases [33-36]. Additionally, researchers have recently developed transgenic calves that produce human immunoglobulin, speaking to the incredible importance of cattle as model organisms for the study of human immunity and disease [37]. Secondly, understanding the molecular and genetic basis of immunity in cattle breeds can not only serve to further our understanding of the breeds, but also to provide genetic information which can be used for selective breeding in order to improve performance and survival of livestock. Immunity in cattle varies vastly by breed. For example, African cattle are known for their incredible resistance to tick and gastrointestinal parasite infestations, traits that have developed in response to thousands of years of evolution in the harsh environments of Africa. A particularly amazing adaptation is the resistance of several African breeds to trypanosomiasis, also known as sleeping sickness [38]. Identification of genes responsible for immunity and introduction of identified immunity-related genes in cattle breeds that are productive but highly susceptible to disease may improve their resistance, survival, and productivity. Understanding genetic features controlling these mechanisms will allow researchers to develop appropriate breeding strategies.

Table 3 Selected immune system-related genes and affiliated protein domains

\begin{tabular}{|c|c|c|c|c|c|c|c|}
\hline Gene name & Length & Source & Accession & Description & Start & Stop & E-value \\
\hline scaffold_2520.g67.t1 & 1075 & Pfam & PF13895 & Immunoglobulin domain & 425 & 491 & $5.40 \mathrm{E}-09$ \\
\hline scaffold_19370.g263.t1 & 508 & Pfam & PF07679 & Immunoglobulin I-set domain & 381 & 454 & 8.30E-07 \\
\hline scaffold_8624.g151.t1 & 512 & Pfam & PF13895 & Immunoglobulin domain & 10 & 73 & $3.90 \mathrm{E}-08$ \\
\hline scaffold_14147.g214.t1 & 159 & Pfam & PF07679 & Immunoglobulin I-set domain & 27 & 112 & $3.40 \mathrm{E}-24$ \\
\hline scaffold_13817.g209.t1 & 758 & Pfam & PF00047 & Immunoglobulin domain & 550 & 628 & $5.10 \mathrm{E}-09$ \\
\hline scaffold_5779.g114.t1 & 142 & Pfam & PF07679 & Immunoglobulin I-set domain & 35 & 70 & $6.20 \mathrm{E}-07$ \\
\hline scaffold_46987.g437.t1 & 460 & Pfam & PF09294 & Interferon-alpha/beta receptor, fibronectin type III & 44 & 142 & 1.20E-17 \\
\hline
\end{tabular}


More generally, research in immunoglobulin genetics is particularly salient for several reasons. Although research into the genetic aspects of and expression of genes related to immunoglobulin has been widely conducted in humans and mice, research in this field is lacking when it comes to livestock breeds, particularly cattle. Information is still needed to complete previous information, including the number of available gene segments and gene families. This kind of information can be used in the future to study and create synthetic recombinant species-specific antibodies, which could be used to treat and prevent infectious diseases.

\section{Domain interactions of Hanwoo-specific genes reveal additional links to immunity}

Additionally, more general consideration of significantly identified protein family domains from the Pfam database provided information needed to further understanding the breed-specific molecular mechanisms of Hanwoo cattle. Table 2 lists highly significantly identified ( $E$ - value $<1 \mathrm{XE}-100)$ Pfam domains. In order to assign meaning and infer the function of these domains, which include several not well understood but highly significant protein domains, we searched for these identified domains within DOMINE (http://domine.utdallas.edu/cgi-bin/Domine), a database of known and predicted protein domain interactions [20, 21]. Among these, several interesting results reveal the genetic intricacies of the Hanwoo genome and its functions.

Several of the most significantly identified protein domains appear to be closely linked with immune system function, further supporting our previous findings. For example, the significantly identified Sema domain $(E$-value $=2.30 \mathrm{E}-117)$ appears to be primarily associated with immune system function. The Sema domain not only forms interactions with the Immunoglobulin domain, but also interacts with the Thrombospondin type 1 (TSP-1) domain, which has been shown to control immune regulation. Thrombospondin, an extremely large multi-domain glycoprotein, is crucial to certain mechanisms related to angiogenesis, cell proliferation, and immune responses [39] such as the chemotactic response to tissue damage and the facilitation of phagocytosis of damaged cells [40-42]. Mice deficient in TSP-1 are more susceptible to inflammation and injury, either as a side effect of drugs or as a result of gene activation [43-46]. Given the strong role of this protein domain in immunity, our identification of this pathway here once again confirms that there are unique functions of immunity at play operating specifically in the Hanwoo genome.

\section{Hanwoo-specific genes linked to muscle and other functions}

Significantly identified protein domains with functions related to energy, fat storage, and muscle function may provide insight into the mechanisms behind Hanwoo cattle's uniquely high percentage of intramuscular fat and fat marbling. For example, LNS2 (Lipin/Ned1/ Smp2) domain, which includes Lipin, was significantly identified $(E$-value $=1.30 \mathrm{E}-104)$ in our data (Table 2). Lipin, encoded by the Lpin1 gene, is a powerful gene which largely controls how the body produces, stores, and uses fat. Mice deficient in Lipin do not develop either diet-induced or genetic obesity [47]. Additionally, enhanced Lipin expression has been shown to promote adiposity in mice [48].

Additionally, the Myosin head (motor domain) protein domain, which is associated with muscle function, was significantly identified $(E$-value $=5.60 \mathrm{E}-207$, Table 2$)$. Myosin is a chief component of myofibril filaments, which are responsible for muscle contraction. Myosin also actively participates in the conversion of ATP chemical energy to mechanical energy through its interaction with Actin [49]. Additionally, the Dynein heavy chain and region D6 of the dynein motor domain and 143-3 protein domain were significantly identified ( $E$-values $=1.50 \mathrm{E}-120,3.60 \mathrm{E}-107$ respectively $)$, both of which are also largely responsible for ATP energy conversion [50-52]. These results suggest that these proteins domains are those which are primarily responsible for providing energy to the muscle and possibly causing the breed-specific high percentage of intramuscular fat that is observed in Hanwoo cattle.

Several of the other identified domains, such as the HAP1 N-terminal conserved region domain, were found to lack interactions with any other domains and their specific roles in cattle have not been well established. As we learn more about these proteins and their functions in the future, we may be able to better interpret these results.

\section{Interpretation of gene ontology terms associated with the entire set of Pfam domains}

As previously discussed, we were able to identify 311 Pfam domains mapping to 168 scaffolds not shared with common cattle. We then filtered that list and kept only the highest hits. Within that short list, we revealed high enrichment for muscle and immunology genes. However, this approach provides a very limited look at our results. Thus, we aimed to further explore Hanwoo-specific domains by analyzing the enrichment of functional categories associated with each individual domain of the entire list. Using Interpro [22], we obtained GO (Gene Ontology) Cellular Component (CC), Molecular Function (MF), and Biological Process (BP) terms for each individual domain [23]. Next, gene ontology results were summarized and visualized with the online tool REVIGO (http://revigo.irb.hr; [24] to better interpret our results. Tables 4, 5, and 6 summarize the BP, CC, and MF GO 
terms, respectively. REVIGO calculates "frequency" and "uniqueness" values, with frequency representing the proportion of the specified GO term within the entire Bos taurus species-specific Uniprot protein annotation database, and uniqueness determining within the inputted list whether a term is an outlier when compared semantically to the list as a whole [24].

Next, using REVIGO's Interactive Graph tool [24] and exporting results into the Cytoscape software package [25], we created a graph-based visualization of the identified terms for each GO category. Figures 3, 4, and 5 display visualizations of $\mathrm{BP}, \mathrm{CC}$, and MF GO terms, respectively. The radius of the bubbles represents the generality of the specified term; a small bubble implies higher specificity. The $p$-value of each GO term is represented by the color shading of each bubble, with darker colors representing higher significance. The edges between the nodes of our graph (GO terms) represent the top 3\% strongest pairwise similarities between terms [24].

The BP GO term visualization (Fig. 3) can be characterized by a large number of un-connected solo terms and shows a large diversity of biological processes being affected, meaning that a large rewiring of functionality is embedded in the new genes acquired by Hanwoo cattle. Note that the most significant term is the most specific, 'centriole replication', which is also connected to the general term 'microtubule-based movement'; dynein (significantly identified from our data) moves along microtubules, so this term may reflect the biological processes responsible for dynein's role in ATP energy conversion. This is quite unique and unexpected, since it signals an important role of cell division [53]. The second group of more significant terms are less specific but all related to transport, particularly 'anion transport',

Table 4 Summary of enriched Gene Ontology (GO) biological process (BP) terms among total identified Pfam protein family domains

\begin{tabular}{|c|c|c|c|c|}
\hline term_ID & description & Frequency $^{a}$ & $\log 10 p$-value & Uniqueness $^{\mathrm{b}}$ \\
\hline GO:0008152 & metabolic process & $62.92 \%$ & -4.2076 & 0.974 \\
\hline GO:0007154 & cell communication & $28.75 \%$ & -5.9208 & 0.866 \\
\hline GO:0006139 & nucleobase-containing compound metabolic process & $28.16 \%$ & -31.0655 & 0.776 \\
\hline GO:0007165 & signal transduction & $26.76 \%$ & -18.9208 & 0.794 \\
\hline GO:0006810 & transport & $19.48 \%$ & -32.6383 & 0.765 \\
\hline GO:0006355 & regulation of transcription, DNA-templated & $14.27 \%$ & -8.9586 & 0.608 \\
\hline GO:0006464 & cellular protein modification process & $14.26 \%$ & -11.7212 & 0.62 \\
\hline GO:0007186 & G-protein coupled receptor signaling pathway & $8.87 \%$ & -25.4318 & 0.803 \\
\hline GO:0006508 & proteolyis & $7.74 \%$ & -32.6778 & 0.741 \\
\hline GO:0006811 & ion transport & $7.05 \%$ & -12.2076 & 0.744 \\
\hline GO:0055114 & oxidation-reduction process & $6.85 \%$ & -11.699 & 0.831 \\
\hline GO:0055085 & transmembrane transport & $6.54 \%$ & -32.6383 & 0.714 \\
\hline GO:0016192 & vesicle-mediated transport & $4.60 \%$ & -30.7447 & 0.785 \\
\hline GO:0006886 & intracelular protein transport & $3.09 \%$ & -30.7447 & 0.792 \\
\hline GO:0016567 & protein ubiquitination & $2.40 \%$ & -14.3468 & 0.673 \\
\hline GO:0006820 & anion transport & $2.07 \%$ & -61.2147 & 0.769 \\
\hline GO:0006457 & protein folding & $1.00 \%$ & -19.9208 & 0.736 \\
\hline GO:0007018 & microtubule-based movement & $1.00 \%$ & -45.6021 & 0.843 \\
\hline GO:0035023 & regulation of Rho protein signal transduction & $0.92 \%$ & -11.1675 & 0.832 \\
\hline GO:0016573 & histone acetylation & $0.60 \%$ & -51.3372 & 0.635 \\
\hline GO:0043401 & steroid hormone mediated signaling pathway & $0.46 \%$ & -28.6383 & 0.839 \\
\hline GO:0045454 & cell redox homeostasis & $0.40 \%$ & -27.0269 & 0.865 \\
\hline GO:0000413 & protein peptidyl-prolyl isomerization & $0.24 \%$ & -19.9208 & 0.709 \\
\hline GO:0006400 & tRNA modification & $0.20 \%$ & -34.7447 & 0.692 \\
\hline GO:0018149 & peptide cross-linking & $0.17 \%$ & -13.4318 & 0.731 \\
\hline GO:0007099 & centriole replication & $0.08 \%$ & -153.347 & 0.832 \\
\hline GO:0009396 & folic acid-containing compound biosynthetic process & $0.05 \%$ & -11.699 & 0.786 \\
\hline
\end{tabular}

${ }^{a}$ Frequency represents the proportion of the specified GO term within the entire Bos Taurus species-specific Uniprot protein annotation database. Higher frequencies represent more general and common terms, while terms with a lower frequency are rare and specific

buniqueness represents whether a term is an outlier when compared semantically to the list as a whole 
Table 5 Summary of enriched Gene Ontology (GO) cellular component (CC) terms among total identified Pfam protein family domains

\begin{tabular}{lllll}
\hline term_ID & description & Frequency $^{\mathrm{a}}$ & $\log ^{10}$-value & Uniqueness $^{\mathrm{b}}$ \\
\hline GO:0005622 & intracellular & $63.18 \%$ & -4.2076 & 0.875 \\
GO:0016020 & membrane & $47.23 \%$ & -5.7959 & 0.872 \\
GO:0016021 & integral component of membrane & $29.77 \%$ & -10.8239 & 0.832 \\
GO:0005634 & nucleus & $27.70 \%$ & -10.6576 & 0.688 \\
GO:0005739 & mitochondrion & $9.22 \%$ & -12.5686 & 0.636 \\
GO:0005740 & mitochondrial envelope & $3.09 \%$ & -32.3565 & 0.576 \\
GO:0031012 & extracellular matrix & $2.00 \%$ & -15 & 0.77 \\
GO:0016459 & myosin complex & $0.38 \%$ & -206.252 & 0.589 \\
GO:0030286 & dynein complex & $0.20 \%$ & -45.6021 & 0.594 \\
\hline
\end{tabular}

${ }^{\mathrm{a}}$ Frequency represents the proportion of the specified GO term within the entire Bos Taurus species-specific Uniprot protein annotation database. Higher frequencies represent more general and common terms, while terms with a lower frequency are rare and specific

${ }^{\mathrm{b}}$ Uniqueness represents whether a term is an outlier when compared semantically to the list as a whole

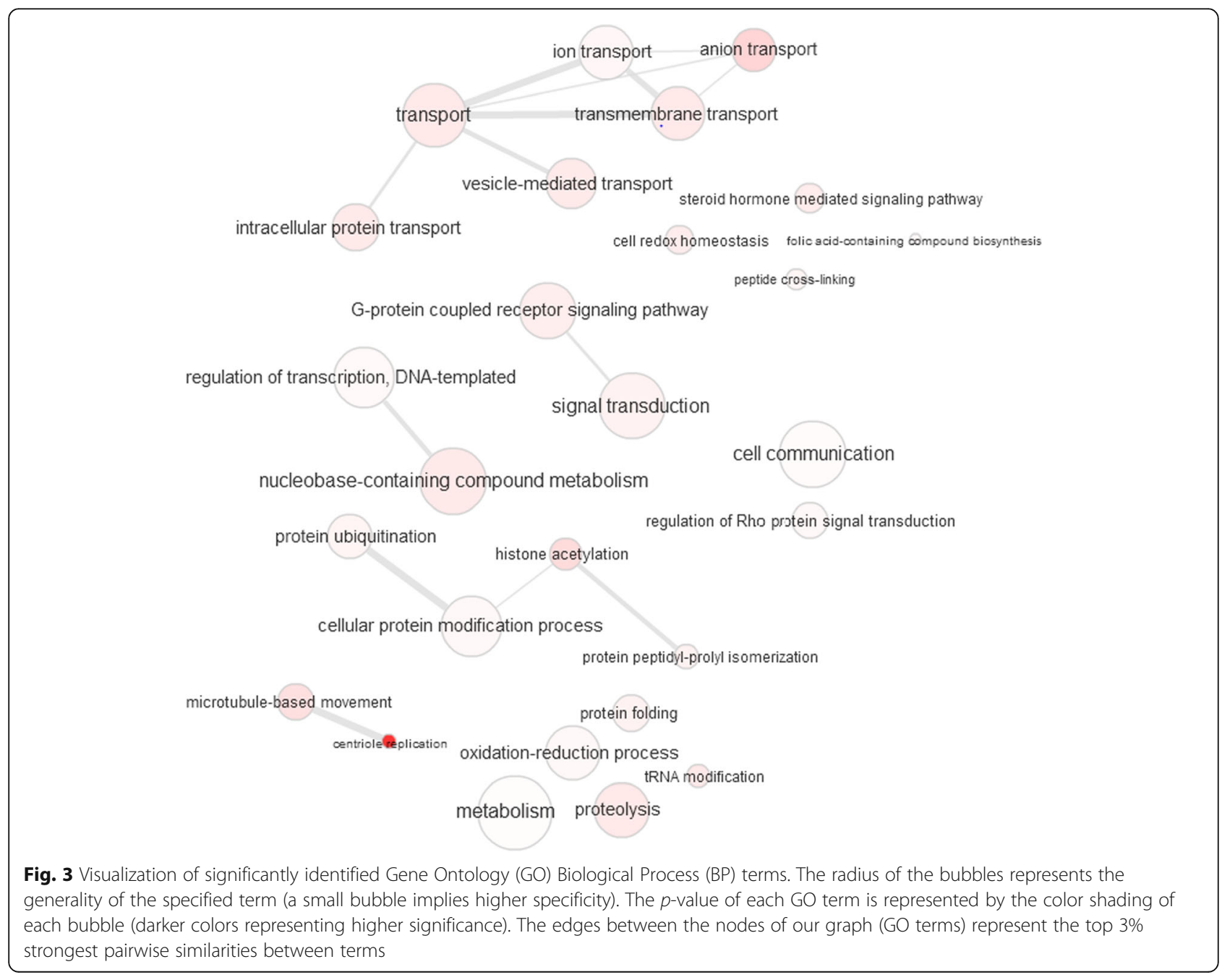


Table 6 Summary of enriched Gene Ontology (GO) molecular function (MF) terms among total identified Pfam protein family domains

\begin{tabular}{|c|c|c|c|c|}
\hline term_ID & description & Frequency $^{a}$ & $\log 10$ p-value & Uniqueness $^{\mathrm{b}}$ \\
\hline GO:0003700 & sequence-specific DNA binding transcription factor activity & $5.30 \%$ & -10.6576 & 0.958 \\
\hline GO:0003712 & transcription cofactor activity & $1.78 \%$ & -39.8861 & 0.957 \\
\hline GO:0003824 & catalytic activity & $37.22 \%$ & -13.4559 & 0.972 \\
\hline GO:0004871 & signal transducer activity & $11.94 \%$ & -25.4318 & 0.927 \\
\hline GO:0004930 & G-protein coupled receptor activity & $7.87 \%$ & -27.6198 & 0.925 \\
\hline GO:0005089 & Rho guanyl-nucleotide exchange factor activity & $0.48 \%$ & -11.699 & 0.956 \\
\hline GO:0005216 & ion channel activity & $2.49 \%$ & -13.9208 & 0.896 \\
\hline GO:0015075 & ion transmembrane transporter activity & $5.28 \%$ & -5.7959 & 0.895 \\
\hline GO:0016773 & phosphotransferase activity, alcohol group as acceptor & $4.60 \%$ & -58.7447 & 0.771 \\
\hline GO:0004672 & protein kinase activity & $3.87 \%$ & -14.1308 & 0.772 \\
\hline GO:0043015 & gamma-tubulin binding & $0.10 \%$ & -25.8539 & 0.878 \\
\hline GO:0008017 & microtubule binding & $1.06 \%$ & -16.4815 & 0.848 \\
\hline GO:0019001 & guanyl nucleotide binding & $2.48 \%$ & -25.4318 & 0.846 \\
\hline GO:0005509 & calcium ion binding & $3.94 \%$ & -19.0655 & 0.863 \\
\hline GO:0005515 & protein binding & $26.71 \%$ & -7.3665 & 0.92 \\
\hline GO:0004488 & methylenetetrahydrofolate dehydrogenase (NADP+) activity & $0.02 \%$ & -13.4559 & 0.878 \\
\hline GO:0003755 & peptidyl-prolyl cis-trans isomerase activity & $0.26 \%$ & -19.9208 & 0.876 \\
\hline GO:0003777 & microtubule motor activity & $0.51 \%$ & -45.6021 & 0.814 \\
\hline GO:0005544 & calcium-dependent phospholipid binding & $0.17 \%$ & -19.0655 & 0.844 \\
\hline GO:0042802 & identical protein binding & $4.77 \%$ & -25.8539 & 0.878 \\
\hline GO:0031683 & G-protein beta/gamma-subunit complex binding & $0.16 \%$ & -25.4318 & 0.883 \\
\hline GO:0043565 & sequence-specific DNA binding & $4.31 \%$ & -10.6576 & 0.855 \\
\hline GO:0016787 & hydrolase activity & $15.05 \%$ & -7.8861 & 0.841 \\
\hline GO:0008484 & sulfuric ester hydrolase activity & $0.11 \%$ & -31.7447 & 0.832 \\
\hline GO:0004181 & metallocarboxypeptidase activity & $0.15 \%$ & -32.6778 & 0.809 \\
\hline GO:0004222 & metalloendopeptidase activity & $0.79 \%$ & -15 & 0.79 \\
\hline GO:0019901 & protein kinase binding & $1.80 \%$ & -7.2007 & 0.885 \\
\hline GO:0008479 & queuine tRNA-ribosyltransferase activity & $0.02 \%$ & -34.7447 & 0.849 \\
\hline GO:0003676 & nucleic acid binding & $21.33 \%$ & -8.6021 & 0.849 \\
\hline GO:0005102 & receptor binding & $6.56 \%$ & -62.1871 & 0.875 \\
\hline GO:0004402 & histone acetyltransferase activity & $0.24 \%$ & -51.3372 & 0.812 \\
\hline GO:0003677 & DNA binding & $10.28 \%$ & -9.5376 & 0.843 \\
\hline GO:0008408 & 3'-5' exonuclease activity & $0.18 \%$ & -31.0655 & 0.827 \\
\hline GO:0016746 & transferase activity, transferring acyl groups & $1.42 \%$ & -30.9208 & 0.805 \\
\hline GO:0003723 & RNA binding & $7.68 \%$ & -5.9208 & 0.847 \\
\hline GO:0046872 & metal ion binding & $20.96 \%$ & -4.5376 & 0.845 \\
\hline GO:0004550 & nucleoside diphosphate kinase activity & $0.14 \%$ & -55.585 & 0.816 \\
\hline GO:0008236 & serine-type peptidase activity & $1.32 \%$ & -7.1739 & 0.793 \\
\hline GO:0008270 & zinc ion binding & $6.73 \%$ & -4.2076 & 0.856 \\
\hline GO:0004129 & cytochrome-c oxidase activity & $0.43 \%$ & -12.5686 & 0.809 \\
\hline GO:0003924 & GTPase activity & $1.03 \%$ & -25.4318 & 0.806 \\
\hline GO:0005524 & ATP binding & $8.83 \%$ & -14.1308 & 0.751 \\
\hline GO:0000166 & nucleotide binding & $14.40 \%$ & -7.3565 & 0.823 \\
\hline GO:0003810 & protein-glutamine gamma-glutamyltransferase activity & $0.07 \%$ & -14.7696 & 0.823 \\
\hline
\end{tabular}


Table 6 Summary of enriched Gene Ontology (GO) molecular function (MF) terms among total identified Pfam protein family domains (Continued)

\begin{tabular}{lllll}
\hline term_ID & description & Frequency $^{\mathrm{a}}$ & $\log _{10}$ p-value & Uniqueness $^{\mathrm{b}}$ \\
\hline GO:0005543 & phospholipid binding & $1.33 \%$ & -16.6021 & 0.829 \\
GO:0035091 & phosphatidylinositol binding & $0.84 \%$ & -17 & 0.829 \\
\hline
\end{tabular}

${ }^{\mathrm{a}}$ Frequency represents the proportion of the specified GO term within the entire Bos Taurus species-specific Uniprot protein annotation database. Higher frequencies represent more general and common terms, while terms with a lower frequency are rare and specific

${ }^{b}$ Uniqueness represents whether a term is an outlier when compared semantically to the list as a whole

which may be associated with ATP energetics. Another uniqueness is the steroid hormone mediated signaling pathway. Sex steroid hormones play a critical role in the regulation of muscle, muscle strength, and growth and maintenance of muscle mass [54]. While identification of this GO term most likely can be attributed to the aforementioned relationship between steroid hormones and muscle development, as a result of the breed-specific unique high-fat muscle development, it may also be due to the practices under which Hanwoo are reared in order to enhance the natural fat marbling in their meat, such as feeding time and diet. For example, cattle are fed a high-concentration grain diet as opposed to grass-feeding [55]. Diet has been shown to have an effect on steroid hormones [56], which may also in part explain the identification of this GO term here.

The CC GO term visualization (Fig. 4) can be characterized by a single connected group consisting of four terms: dynein complex, myosin complex, mitochondrial envelope, and mitochondrion. As previously mentioned, the Myosin Head and Dynein heavy chain protein domains were found significantly identified in our resultsboth of which participate in the conversion of ATP chemical energy to mechanical energy and serve crucial functions for muscle function. The connectivity of these nodes within our network visualization signifies that these two components work together and are potentially significant in Hanwoo-specific characteristics, such as their high percentage of intramuscular fat. The rest of the terms are generic, independent $\mathrm{CC}$ terms that include nucleus and membrane.

The MF GO term visualization (Fig. 5) can be characterized by high connectivity, with the most significant values grouped together. Microtubule motor activity, another microtubule function related term, was also identified at the molecular function level, once again suggesting ATP energetics at play. A unique feature of this visualization, compared to the $\mathrm{BP}$ and $\mathrm{CC}$ visualizations, is the presence of 4 unconnected graphs as opposed to many unconnected terms or a single connected group. The first group features solely terms related to binding. This group contains the following terms: Sequence-specific DNA binding, DNA binding, RNA binding, Nucleic acid binding, ATP binding, Phospholipid binding, Calcium-dependent phospholipid binding, Guanyl nucleotide binding, Metal ion binding, Zinc ion binding, and Calcium ion binding. The second

dynein complex

myosin complex

mitochondrial envelope

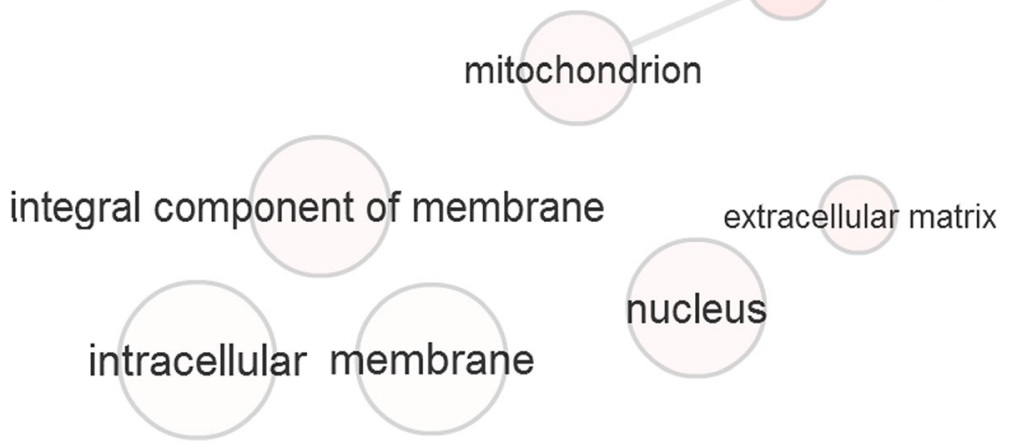

Fig. 4 Visualization of significantly identified Gene Ontology (GO) Cellular Component (CC) terms. The radius of the bubbles represents the generality of the specified term (a small bubble implies higher specificity). The p-value of each GO term is represented by the color shading of each bubble (darker colors representing higher significance). The edges between the nodes of our graph (GO terms) represent the top 3\% strongest pairwise similarities between terms 


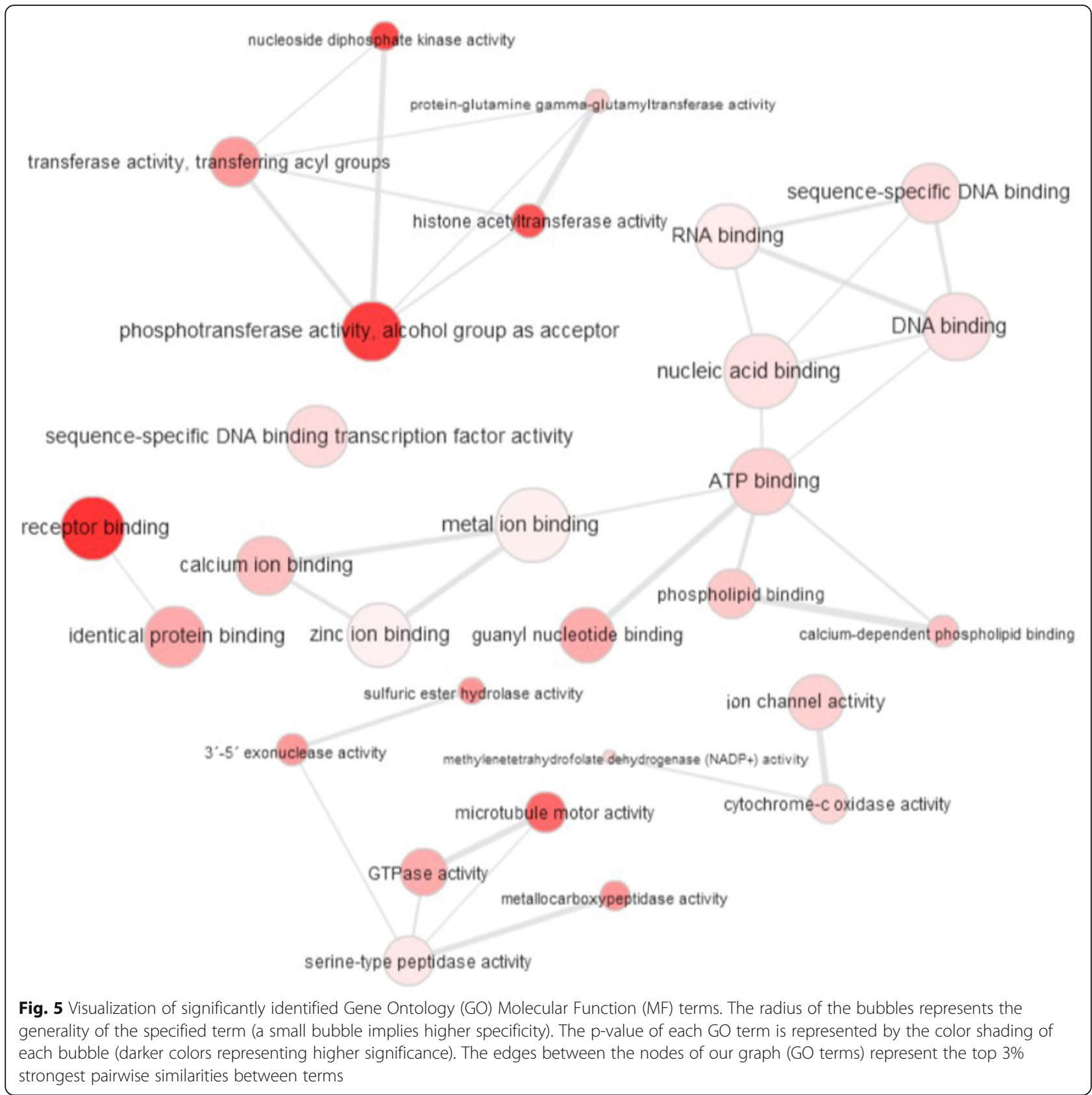

group consists of three connected terms: Ion Channel activity, Methylenetetrahydrofolate dehydrogenase (NADP+) activity, and Cytochrome-c oxidase activity. The third group consists of six connected terms: Sulfuric ester hydrolase activity, $3^{\prime}-5^{\prime}$ exonuclease activity, Microtubule motor activity, GTPase activity, Serine-type peptidase activity, and Metallocarboxypeptidase activity.

The fourth and final group consists of 5 terms related to the activity of transferases: Nucleoside diphosphate kinase activity, Transferase activity, transferring acyl groups, Protein-glutamine gamma-glutamyltransferase activity, Histone acetyltransferase activity, and Phosphotransferase activity, alcohol group as acceptor. Transferases are enzymes which are responsible for catalyzation of the transfer of certain functional groups from one molecule to another. They are essential for countless biochemical processes throughout the body. In cattle specifically, it has been shown that the activity of transferases is critical for embryo development [57]. The expression of genes with transferase activity function varies between abnormal and normal pregnancies $[58,59]$. Therefore, the expression of these transferase GO terms may be due to their role in healthy pregnancy and development. However, interestingly, results of previous studies have demonstrated a 
correlation between certain transferase activity genes, such as GPAT1 and ATGL, and intramuscular fat content in Korean Cattle [60]. These previously identified results, when taken along with the comparatively high expression and connectivity of $\mathrm{GO}$ terms related to transferase activity, suggests that there may be unique mechanisms of transferase activity in Hanwoo cattle which influences their development and may perhaps be a factor impacting their species-specific high percentage of intramuscular fat.

\section{Conclusions}

The information unearthed from the comparison of breeds and identification of genetic variation in this study will be invaluable for future research on the molecular determinants that have been bred in Hanwoo cattle. Results revealed Hanwoo-specific protein domains which were largely characterized by immunoglobulin function. Furthermore, domain interactions of Hanwoo-specific genes reveal additional links to immunity. Hanwoo-specific genes linked to muscle and other functions were identified, including protein domains with functions related to energy, fat storage, and muscle function that may provide insight into the mechanisms behind Hanwoo cattle's uniquely high percentage of intramuscular fat and fat marbling. Analyzing the whole Hanwoo genome and reporting significant genomic variations is crucial to identifying genetic novelties that are arising from useful adaptations. Similarly, such analysis will allow future researchers to compare and classify breeds, identify important genetic markers, and develop breeding strategies to further improve traits of economic value and biological significance.

\section{Additional files}

Additional file 1: Table S1. Summary of sequencing data (DOCX $28 \mathrm{~kb}$ ) Additional file 2: Table S2. Significantly identified ( $E$ - value $<1 X E-40$ ) Pfam protein family domain analysis results. (DOCX $17 \mathrm{~kb}$ )

Additional file 3: FASTA sequences for scaffolds which have locations with depth > 10x. (XLSX 9907 kb)

Additional file 4: Protein sequences which have locations with depth > 10X. (XLSX $101 \mathrm{~kb})$

\section{Abbreviations}

BP: Biological process; CC: Cellular component; GO: Gene ontology; MF: Molecular function

\section{Acknowledgements}

We acknowledge the support from different institutions and their personnel providing help for the sampling of cattle (Hanwoo Improvement Center of the National Agricultural Cooperative Federation, HICNACF) and cattle keepers for their assistance and permission to sample their herds.

\section{Funding}

This work was supported by Agenda (PJ01251902) of the National Institute of Animal Science, Rural Development Administration (RDA), Republic of Korea.

\section{Availability of data and materials}

All information supporting the results of this manuscript are included within the article and additional files. The sequences of genes predicted through assembly of unaligned reads have been uploaded as a Additional files 1, 2, 3 and 4.

\section{Authors' contributions}

KCA conceived the project and designed scientific objectives. DL performed Hanwoo sample collection and data generation. $\mathrm{BHC}$ conducted experimental design of the Hanwoo population. WK, SS, and KK performed genome annotation and data analysis. KCA performed comparative analysis using bioinformatics tools. KCA interpreted data and wrote the manuscript. HK and DL organized and supervised the project. All authors read and approved the final manuscript.

Ethics approval and consent to participate

No ethics statement was required for the collection of DNA samples. DNA was extracted either from artificial insemination bull semen straws or from blood samples obtained from the Hanwoo Improvement Center of the National Agricultural Cooperative Federation (HICNACF) with the permission of the owners. The protocol was approved by the Committee on the Ethics of Animal Experiments of the National Institute of Animal Science (Permit Number: NIAS2015-774).

\section{Competing interests}

The authors declare that they have no competing interests.

\section{Publisher's Note}

Springer Nature remains neutral with regard to jurisdictional claims in published maps and institutional affiliations.

\section{Author details}

${ }^{1}$ Interdisciplinary Program in Bioinformatics, Seoul National University, Kwan-ak St. 599, Kwan-ak Gu, Seoul 151-741, Republic of Korea. ${ }^{2}$ Department of Agricultural Biotechnology and Research Institute for Agriculture and Life Sciences, Seoul National University, Seoul 151-921, Republic of Korea. ${ }^{3} \mathrm{CHO \& KIM}$ genomics, Main Bldg. \#514, SNU Research Park, Seoul National University Mt.4-2, NakSeoungDae, Gwanakgu, Seoul 151-919, Republic of Korea. ${ }^{4}$ Animal Genomics \& Bioinformatics Division, National Institute of Animal Science, RDA, 77 Chuksan-gil, Kwonsun-gu, Suwon 441-706, Republic of Korea.

Received: 15 November 2017 Accepted: 14 May 2018

Published online: 29 May 2018

\section{References}

1. Lee S-H, Park B-H, Sharma A, Dang C-G, Lee S-S, Choi T-J, Choy Y-H, Kim HC, Jeon K-J, Kim S-D, et al. Hanwoo cattle: origin, domestication, breeding strategies and genomic selection. J Anim Sci Technol. 2014;56(1):2.

2. Jo C, Cho SH, Chang J, Nam KC. Keys to production and processing of Hanwoo beef: a perspective of tradition and science. Anim Front. 2012;2(4):32-8.

3. Kim HJ, Sharma A, Lee SH, Lee DH, Lim DJ, Cho YM, Yang BS, Lee SH. Genetic association of PLAG1, SCD, CYP7B1 and FASN SNPS and their effects on carcass weight, intramuscular fat and fatty acid composition in Hanwoo steers (Korean cattle). Anim Genet. 2016;48. https://doi.org/10.1111/age. 12523.

4. Hwang YH, Joo ST. Fatty acid profiles of ten muscles from high and low marbled (quality grade 1++ and 2) Hanwoo steers. Korean J Food Sci Anim Resour. 2016;36(5):679-88.

5. Sudrajad P, Sharma A, Dang CG, Kim JJ, Kim KS, Lee JH, Kim S, Lee SH. Validation of single nucleotide polymorphisms associated with carcass traits in a commercial Hanwoo population. Asian Australas J Anim Sci. 2016; 29(11):1541-6.

6. Li XZ, Park BK, Hong BC, Ahn JS, Shin JS. Effect of soy lecithin on total cholesterol content, fatty acid composition and carcass characteristics in the Longissimus dorsi of Hanwoo steers (Korean native cattle). Anim Sci J. 2016;

7. Cho SH, Kang G, Seong P, Kang S, Sun C, Jang S, Cheong JH, Park B, Hwang I. Meat quality traits as a function of cow maturity. Anim Sci J. 2016;

8. Andrews S: FastQC: a quality control tool for high throughput sequence data. Reference Source 2010.

9. Bolger AM, Lohse M, Usadel B. Trimmomatic: a flexible trimmer for Illumina sequence data. Bioinformatics 2014:30(15):2114-20. 
10. Langmead B, Salzberg SL. Fast gapped-read alignment with bowtie 2. Nat Methods. 2012;9(4):357-9.

11. Li H, Handsaker B, Wysoker A, Fennell T, Ruan J, Homer N, Marth G, Abecasis G, Durbin R. Genome project data processing S: the sequence alignment/ map format and SAMtools. Bioinformatics. 2009;25(16):2078-9.

12. McKenna A, Hanna M, Banks E, Sivachenko A, Cibulskis K, Kernytsky A, Garimella K, Altshuler D, Gabriel S, Daly M, et al. The genome analysis toolkit: a MapReduce framework for analyzing next-generation DNA sequencing data. Genome Res. 2010;20(9):1297-303.

13. DePristo MA, Banks E, Poplin R, Garimella KV, Maguire JR, Hartl C, Philippakis AA, del Angel G, Rivas MA, Hanna M, et al. A framework for variation discovery and genotyping using next-generation DNA sequencing data. Nat Genet. 2011;43(5):491-8.

14. Van der Auwera GA, Carneiro MO, Hartl C, Poplin R, Del Angel G, LevyMoonshine A, Jordan T, Shakir K, Roazen D, Thibault J et al: From FastQ data to high confidence variant calls: the genome analysis toolkit best practices pipeline. Curr Protoc Bioinformatics 2013, 43:11.10.11-11.10.33.

15. Gnerre S, MacCallum I, Przybylski D, Ribeiro FJ, Burton JN, Walker BJ, Sharpe T, Hall G, Shea TP, Sykes S, et al. High-quality draft assemblies of mammalian genomes from massively parallel sequence data. Proc Natl Acad Sci. 2011; 108(4):1513-8

16. Ribeiro FJ, Przybylski D, Yin S, Sharpe T, Gnerre S, Abouelleil A, Berlin AM, Montmayeur A, Shea TP, Walker BJ, et al. Finished bacterial genomes from shotgun sequence data. Genome Res. 2012;22(11):2270-7.

17. Peng Y, Leung HCM, Yiu SM, Chin FYL. IDBA - a practical iterative de Bruijn graph De novo assembler. In: Berlin BB, editor. Research in computational molecular biology: 14th annual international conference, RECOMB 2010, Lisbon, Portugal, April 25-28, 2010 proceedings. Heidelberg: Springer Berlin Heidelberg; 2010. p. 426-40.

18. Peng Y, Leung HC, Yiu SM, Chin FY. IDBA-UD: a de novo assembler for single-cell and metagenomic sequencing data with highly uneven depth. Bioinformatics. 2012;28(11):1420-8.

19. Finn RD, Coggill P, Eberhardt RY, Eddy SR, Mistry J, Mitchell AL, Potter SC, Punta M, Qureshi M, Sangrador-Vegas A, et al. The Pfam protein families database: towards a more sustainable future. Nucleic Acids Res. 2016:44(D1):D279-85

20. Raghavachari B, Tasneem A, Przytycka TM, Jothi R. DOMINE: a database of protein domain interactions. Nucleic Acids Res. 2008;36(Database issue): D656-61.

21. Yellaboina S, Tasneem A, Zaykin DV, Raghavachari B, Jothi R. DOMINE: a comprehensive collection of known and predicted domain-domain interactions. Nucleic Acids Res. 2011;39(Database issue):D730-5.

22. Finn RD, Attwood TK, Babbitt PC, Bateman A, Bork P, Bridge AJ, Chang H-Y, Dosztányi Z, El-Gebali S, Fraser M, et al. InterPro in 2017-beyond protein family and domain annotations. Nucleic Acids Res. 2017;45(D1):D190-9.

23. Ashburner M, Ball CA, Blake JA, Botstein D, Butler H, Cherry JM, Davis AP, Dolinski K, Dwight SS, Eppig JT, et al. Gene ontology: tool for the unification of biology. The gene ontology consortium. Nat Genet. 2000;25(1):25-9.

24. Supek F, Bošnjak M, Škunca N, Šmuc T. REVIGO summarizes and visualizes long lists of gene ontology terms. PLoS One. 2011;6(7):e21800.

25. Killcoyne S, Carter GW, Smith J, Boyle J. Cytoscape: a community-based framework for network modeling. Methods Mol Bio. 2009;563:219-39.

26. Pestka S, Langer JA, Zoon KC, Samuel CE. Interferons and their actions. Annu Rev Biochem. 1987;56:727-77.

27. Balkwill F. Interferons and other regulatory cytokines. Immunology. 1989; 66(4):634.

28. Roberts RM. Interferon-tau, a type 1 interferon involved in maternal recognition of pregnancy. Cytokine Growth Factor Rev. 2007;18(5-6):403-8.

29. Han CS, Mathialagan N, Klemann SW, Roberts RM. Molecular cloning of ovine and bovine type I interferon receptor subunits from uteri, and endometrial expression of messenger ribonucleic acid for ovine receptors during the estrous cycle and pregnancy. Endocrinology. 1997; 138(11):4757-67.

30. Platanias LC. Mechanisms of type--- and type-II-interferon-mediated signalling. Nat Rev Immunol. 2005;5(5):375-86.

31. de Souza JAC, Rossa Junior C, Garlet GP, Nogueira AVB, Cirelli JA. Modulation of host cell signaling pathways as a therapeutic approach in periodontal disease. J Appl Oral Sci. 2012;20(2):128-38.

32. Zhao Y, Kacskovics I, Rabbani H, Hammarstrom L. Physical mapping of the bovine immunoglobulin heavy chain constant region gene locus. J Biol Chem. 2003;278(37):35024-32.
33. Casswall TH, Nilsson HO, Bjorck L, Sjostedt S, Xu L, Nord CK, Boren T, Wadstrom T, Hammarstrom L. Bovine anti-helicobacter pylori antibodies for oral immunotherapy. Scand J Gastroenterol. 2002;37(12):1380-5.

34. Hammarstrom L, Gardulf A, Hammarstrom V, Janson A, Lindberg K, Smith Cl. Systemic and topical immunoglobulin treatment in immunocompromised patients. Immunol Rev. 1994;139:43-70.

35. Weiner C, Pan Q, Hurtig M, Boren T, Bostwick E, Hammarstrom L. Passive immunity against human pathogens using bovine antibodies. Clin Exp Immunol. 1999;116(2):193-205.

36. Lilius EM, Marnila P. The role of colostral antibodies in prevention of microbial infections. Curr Opin Infect Dis. 2001;14(3):295-300.

37. Kuroiwa Y, Kasinathan P, Choi YJ, Naeem R, Tomizuka K, Sullivan EJ, Knott JG, Duteau A, Goldsby RA, Osborne BA, et al. Cloned transchromosomic calves producing human immunoglobulin. Nat Biotechnol. 2002;20(9):889-94.

38. Roelants GE, Fumoux F, Pinder M, Queval R, Bassinga A, Authie E. Identification and selection of cattle naturally resistant to African trypanosomiasis. Acta Trop. 1987;44(1):55-66.

39. Lopez-Dee Z, Pidcock K, Gutierrez LS. Thrombospondin-1: multiple paths to inflammation. Mediat Inflamm. 2011:2011:296069.

40. Wight TN, Raugi GJ, Mumby SM, Bornstein P. Light microscopic immunolocation of thrombospondin in human tissues. J Histochem Cytochem. 1985;33(4):295-302.

41. Grimbert P, Bouguermouh S, Baba N, Nakajima T, Allakhverdi Z, Braun D, Saito H, Rubio M, Delespesse G, Sarfati M. Thrombospondin/CD47 interaction: a pathway to generate regulatory $T$ cells from human CD4+ CD25- T cells in response to inflammation. J Immunol. 2006;177(6):3534-41.

42. Doyen V, Rubio M, Braun D, Nakajima T, Abe J, Saito H, Delespesse G, Sarfati $M$. Thrombospondin 1 is an Autocrine negative regulator of human dendritic cell activation. J Exp Med. 2003;198(8):1277-83.

43. Contreras-Ruiz L, Regenfuss B, Mir FA, Kearns J, Masli S. Conjunctival inflammation in thrombospondin-1 deficient mouse model of Sjogren's syndrome. PLoS One. 2013;8(9):e75937.

44. Ezzie ME, Piper MG, Montague C, Newland CA, Opalek JM, Baran C, Ali N, Brigstock D, Lawler J, Marsh CB. Thrombospondin-1-deficient mice are not protected from bleomycin-induced pulmonary fibrosis. Am J Respir Cell Mol Biol. 2011:44(4):556-61.

45. Zhao Y, Xiong Z, Lechner EJ, Klenotic PA, Hamburg BJ, Hulver M, Khare A, Oriss T, Mangalmurti N, Chan Y, et al. Thrombospondin-1 triggers macrophage IL-10 production and promotes resolution of experimental lung injury. Mucosal Immunol. 2014;7(2):440-8.

46. Punekar S, Zak S, Kalter VG, Dobransky L, Punekar I, Lawler JW, Gutierrez LS. Thrombospondin 1 and its mimetic peptide ABT-510 decrease angiogenesis and inflammation in a murine model of inflammatory bowel disease. Pathobiology. 2008;75(1):9-21.

47. Phan J, Peterfy M, Reue K. Lipin expression preceding peroxisome proliferator-activated receptor-gamma is critical for adipogenesis in vivo and in vitro. J Biol Chem. 2004;279(28):29558-64.

48. Phan J, Reue K. Lipin, a lipodystrophy and obesity gene. Cell Metab. 2005; 1(1):73-83.

49. Rayment I, Holden HM, Whittaker M, Yohn CB, Lorenz M, Holmes KC, Milligan RA. Structure of the actin-myosin complex and its implications for muscle contraction. Science. 1993;261(5117):58-65.

50. Roberts AJ. Functions and mechanics of dynein motor. Proteins. 2013;14(11): $713-26$.

51. Bunney TD, van Walraven $\mathrm{HS}$, de Boer AH. 14-3-3 protein is a regulator of the mitochondrial and chloroplast ATP synthase. Proc Natl Acad Sci. 2001; 98(7):4249-54

52. Berg D, Holzmann C, Riess O. 14-3-3 proteins in the nervous system. Nat Rev Neurosci. 2003;4(9):752-62

53. Nigg EA, Stearns T. The centrosome cycle: centriole biogenesis, duplication and inherent asymmetries. Nat Cell Biol. 2011;13(10):1154-60.

54. McClung JM, Davis JM, Wilson MA, Goldsmith EC, Carson JA. Estrogen status and skeletal muscle recovery from disuse atrophy. J Appl Physiol. 2006; 100(6):2012-23.

55. Gotoh T, Joo S-T. Characteristics and health benefit of highly marbled Wagyu and Hanwoo beef. Korean J Food Sci Anim Resour. 2016;36(6):709-18.

56. Chesworth JM, Easdon MP. Effect of diet and season on steroid hormones in the ruminant. J Steroid Biochem. 1983;19(1c):715-23.

57. Adams HA, Southey BR, Everts RE, Marjani SL, Tian CX, Lewin HA, Rodriguez-Zas SL. Transferase activity function and system development process are critical in cattle embryo development. Funct Integr Genomics. 2011;11(1):139-50. 
58. Mirlesse $V$, Jacquemard F, Daffos F, Forestier F. Fetal gammaglutamyl transferase activity: clinical implication in fetal medicine. Biol Neonate. 1996; 70(4):193-8

59. Gibbs DA, McFadyen IR, Crawfurd MD, De Muinck Keizer EE, HeadhouseBenson CM, Wilson TM, Farrant PH. First-trimester diagnosis of Lesch-Nyhan syndrome. Lancet. 1984;2(8413):1180-3.

60. Jeong J, Kwon EG, Im SK, Seo KS, Baik M. Expression of fat deposition and fat removal genes is associated with intramuscular fat content in longissimus dorsi muscle of Korean cattle steers. J Anim Sci. 2012;90(6):2044-53.

Ready to submit your research? Choose BMC and benefit from:

- fast, convenient online submission

- thorough peer review by experienced researchers in your field

- rapid publication on acceptance

- support for research data, including large and complex data types

- gold Open Access which fosters wider collaboration and increased citations

- maximum visibility for your research: over $100 \mathrm{M}$ website views per year 